\title{
Manejo y tratamiento de pacientes en áreas quirúrgicas durante la pandemia COVID-19 Revisión en el área de Ortopedia y Traumatología
}

\author{
Andrés Cattáneo, Esteban F. Coppola, A. Federico Medina, Alejandro Pachón Guzmán \\ Hospital Zonal General de Agudos "Mi Pueblo", Florencio Varela, Buenos Aires - Argentina
}

\begin{abstract}
RESUMEN
La pandemia actual ocasionada por el coronavirus (COVID-19) ha promovido el desarrollo de gran cantidad de medidas en el ámbito individual y global, que afectan gravemente a los sistemas de salud. Aunque la epidemia no involucra directamente a los especialistas en Ortopedia y Traumatología, indirectamente, nos vemos compelidos a implementar medidas hospitalarias preventivas tendientes a disminuir las posibilidades de contagio durante nuestros procedimientos específicos. Esta breve revisión bibliográfica pretende aportar información dinámica básica respecto a de qué manera actuar como especialistas en las áreas quirúrgicas ante casos sospechosos/confirmados de COVID-19. Promovemos el diseño de planes de trabajo para la atención de pacientes antes de la cirugía, durante esta y después.
\end{abstract}

Palabras clave: Coronavirus; COVID-19; quirófano; cirugías; Ortopedia y Traumatología; pandemia.

Management and treatment of patients in the surgical area during COVID-19 pandemic.

Orthopedic literature review

\section{ABSTRACT}

The current coronavirus (COVID-19) pandemic has led to a large number of measures at the individual and global level, seriously affecting healthcare systems. Although COVID-19 does not directly involve specialists in Orthopedics, we are nevertheless compelled to implement preventive hospital measures aimed at reducing chances of infection during our specific procedures. This literature review aims to provide basic dynamic information on how healthcare specialists should act in surgical areas when facing suspicious/confirmed cases of COVID-19. We advocate the design of updated workflow processes for patient care before, during and after surgery.

Key words: Coronavirus; COVID-19; operating room; surgery; Orthopedics; pandemic.

\section{INTRODUCCIÓN}

Los eventos ocasionados por la pandemia del coronavirus 19 (SARS-CoV-2) afectan y exigen la capacidad máxima de los sistemas sanitarios por su rápida expansión mundial. Esto obliga a implementar cambios en los algoritmos de manejo y circulación de los pacientes, en el ámbito hospitalario, para responder, de manera eficiente y segura, a las demandas que la pandemia genere. Dichas demandas incluyen el requerimiento de procedimientos quirúrgicos en los servicios de emergencias ante cuadros que así lo requieran, en pacientes con sospecha o confirmación de COVID-19. En función de lo expuesto y como integrantes del Servicio de Ortopedia y Traumatología del Hospital Zonal General de Agudos "Mi Pueblo" de Florencio Varela, decidimos hacer un relevamiento de la información científica en PubMed, revistas digitales europeas de especialidades médico-quirúrgicas (AO Foundation, Sociedad Española de Cirugía Ortopédica y Traumatología, Asociación Argentina de Ortopedia y Traumatología) y páginas de organizaciones de salud relevantes (Organización Mundial de la Salud [OMS], Organización Panamericana de la Salud, Centers for Disease Control and Prevention). Se utilizaron las palabras clave: "covid-19", "coronavirus", "cirugía”, "surgery", "estrés quirúrgico". El objetivo de esta búsqueda fue proponer

Recibido el 14-4-2020. Aceptado luego de la evaluación el 15-4-2020. Dr. ALEJANDRO PACHÓN GUZMÁN • apachonguzman@ gmail.com

Cómo citar este artículo: Cattáneo A, Coppola EF, Medina AF, Pachón Guzmán A. Manejo y tratamiento de pacientes en áreas quirúrgicas durante la pandemia COVID-19. Revisión en el área de Ortopedia y Traumatología. Rev Asoc Argent Ortop Traumatol 2020;85(2):174-183. https://doi.org/10.15417/issn.1852-7434.2020.85.2.1101. 
un protocolo institucional que brinde pautas de comportamiento para el equipo profesional, en el ámbito prehospitalario e intrahospitalario, así como también, en el seguimiento posoperatorio. De este modo, pretendemos disminuir el impacto de la enfermedad y su posible transmisión tanto a otros pacientes, como a los integrantes del equipo de salud interviniente, con el fin de prevenir la contaminación de espacios limpios del hospital. Para ello, se relevaron artículos de países del hemisferio norte que debieron modificar sus protocolos anticipadamente, debido al origen y la distribución de la pandemia desde su inicio. Como el especialista en Ortopedia y Traumatología se dedica fundamentalmente a resolver patologías musculoesqueléticas, su actividad no ha sido considerada esencial en la primera línea para el control de una infección viral. Sin embargo, consideramos que, en la coyuntura actual, tiene un papel esencial ayudando a controlar la pandemia y atendiendo a pacientes que requieran la intervención del equipo en emergencia. Por eso, cumpliendo ese rol, debe involucrarse en el trabajo conjunto e interdisciplinario institucional organizado para mitigar el impacto de la pandemia del COVID-19, pero empleando las medidas pertinentes de bioseguridad.

En la práctica médica diaria, se debe poner énfasis en la adopción de medidas que apunten a disminuir, al mínimo posible, las situaciones de contagio. Esto implica introducir cambios en cuanto al contacto con pacientes que requieran un procedimiento urgente o programado impostergable. Estos procedimientos son esenciales para el control de daños en las lesiones traumatológicas graves, generalmente ocasionadas por traumatismos de alta energía con compromiso de estructuras osteoarticulares y de partes blandas circundantes, como fracturas articulares, fracturas expuestas y abiertas, síndrome compartimental, luxaciones de grandes articulaciones, lesiones tendinosas completas o parciales, o lesiones tegumentarias con pérdida de sustancia y exposición de estructuras nobles (tendones, nervios y vasos sanguíneos); intervenciones de procesos infecciosos osteoarticulares graves, como artritis séptica, osteomielitis activa, infecciones graves de extremidades que requieran intervención y procesos tumorales malignos relacionados con la especialidad (asociados o no a fracturas patológicas), ya que si no se tratan pueden dejar secuelas graves y ponen en riesgo la pérdida de la extremidad o el estado general.

\section{RESULTADOS DEL RELEVAMIENTO DE INFORMACIÓN}

\section{Generalidades del virus y su cuadro clínico}

Los coronavirus pertenecen a una amplia familia de virus que provocan infecciones, en general, del aparato respiratorio, que varían desde el resfriado común hasta enfermedades graves, como el síndrome respiratorio agudo severo (SARS-CoV).

Esta nueva cepa SARS-CoV-2, que causa el brote actual del COVID-19, se identificó en diciembre de 2019, en Wuhan, ciudad capital de la provincia de Hubei, en la República Popular China. Para enero de 2020, el brote era considerado una emergencia de salud pública de interés internacional y, en marzo de 2020, fue declarado pandemia por la OMS. ${ }^{1}$

La infección por el SARS-CoV-2, en general, provoca fiebre y síntomas respiratorios, como tos, disnea y odinofagia. El cuadro clínico puede incluir un espectro de signosintomatología de presentación leve, con síntomas gastrointestinales, conjuntivitis, rinorrea, anosmia, mialgia y fatiga. En algunos casos, puede adquirir características graves y provocar cuadros de neumonía y dificultad respiratoria del tipo síndrome respiratorio agudo severo (SARS), insuficiencia renal aguda y causar la muerte en los casos más graves. ${ }^{2} \mathrm{El}$ contagio con el SARS-CoV-2 ocurre, al igual que con otros virus y gripes, por contacto de persona a persona, con gotas respiratorias de la tos o los estornudos. La evidencia demuestra una alta tasa de contagio, inclusive durante el período de incubación, lo que hace muy difícil prevenir el contagio y detectar precozmente los casos positivos. Un porcentaje de pacientes contagiados por coronavirus 19 se ha mantenido asintomático por lapsos mayores o ha desarrollado síntomas menores. ${ }^{3,4}$

\section{RECOMENDACIONES GENERALES PARA PREVENIR EL CONTAGIO}

Las medidas generales recomendadas por la OMS para evitar el contagio del coronavirus 19 incluyen:

- Realizar una buena y frecuente higiene de manos con agua y jabón.

- Usar soluciones desinfectantes, como el alcohol en gel.

- Mantener medidas de higiene respiratoria, como cubrirse la boca y la nariz al toser o estornudar.

- Evitar el contacto estrecho con cualquier persona que tenga síntomas respiratorios agudos.

- Evitar tocarse los ojos, la nariz y la boca.

- Mantener el distanciamiento social. 
En el ámbito hospitalario, las recomendaciones para el personal sanitario son:

- Aumentar las medidas de protección en los distintos niveles de atención desde la primera consulta en la sala de urgencias.

- Usar un equipo de protección personal (EPP) adecuado para la evaluación y el tratamiento de pacientes con COVID-19.

- Extremar las medidas de bioseguridad en los pases de sala.

- Evitar compartir espacios comunitarios entre profesionales.

- Evitar, en lo posible, internaciones prolongadas.

\section{IMPACTO EN LA COMUNIDAD QUIRÚRGICA}

La rapidez con la que la epidemia ha avanzado en el mundo ha promovido la implementación de cambios determinantes en la actividad quirúrgica habitual. Estos cambios incluyen la cancelación de cirugías programadas, la redistribución de los insumos, la baja disponibilidad de componentes hematológicos, la ocupación de profesionales de áreas quirúrgicas en zonas de atención ambulatoria, el uso de espacios quirúrgicos para la internación de pacientes con COVID-19, la dificultad para el seguimiento de los pacientes operados y el aumento de las medidas de bioseguridad durante las cirugías.

El incremento de casos positivos asociado a la necesidad del uso de recursos de soporte vital avanzado y de personal altamente calificado, como los especialistas en terapia intensiva y anestesiología, para el manejo de máquinas de respiración asistida, disminuye la disponibilidad de estos servicios a nivel quirúrgico.

La baja cantidad de donantes con la consiguiente escasa disponibilidad de componentes hematológicos requiere priorizar qué tipo de cirugías se realizarán para disminuir su requerimiento inmediato, siempre que el cuadro clínico lo permita. Por ejemplo, en pacientes con enfermedades oncológicas, iniciar el tratamiento con quimioterapia neoadyuvante, definir el tamaño de la tumoración, de manera precisa, mediante estudios diagnósticos por imágenes y realizar, luego, el procedimiento de manera planificada, y así evitar la exposición del paciente y el requerimiento inmediato de recursos hospitalarios.

Las limitaciones a la circulación de personas han disminuido significativamente las lesiones por accidentes de tránsito. En tal sentido, en el norte de Italia, se decidió reorganizar al personal médico dedicado a la patología traumatológica en tres hospitales de Nivel I para la contención de estos pacientes, sin mezclarlos con casos sospechosos de COVID-19.5

\section{RECOMENDACIONES ESPECÍFICAS PARA EL ÁREA QUIRÚRGICA DESTINADAS A PREVENIR EL CONTAGIO}

Para el desarrollo de la actividad quirúrgica, se aconseja realizar solo procedimientos que se consideren como urgencias y emergencias que, si no se trataran, ocasionarían secuelas permanentes en el paciente. Estas restricciones buscan disminuir los posibles contagios del personal de la salud, el uso de materiales sanitarios que serían fundamentales para las medidas de control de la pandemia y la reducción de la morbimortalidad en pacientes no contagiados con el coronavirus $19,{ }^{6}$ dado que el procedimiento quirúrgico puede disminuir per se la inmunidad celular de manera inmediata. Este mecanismo celular resulta indispensable para el control de las infecciones virales. ${ }^{7}$

El proceso quirúrgico involucra alterar las barreras naturales del organismo y la modificación de la integridad anatómica; esto genera una combinación de impulsos nerviosos aferentes somáticos y autonómicos que producen una respuesta endocrina, en simultáneo con una respuesta inmunitaria e inflamatoria mediada por citoquinas y hormonas consecutiva a la exposición de moléculas intracelulares y la secreción de células endoteliales, leucocitos activados y fibroblastos. Este desequilibrio se conoce como estrés quirúrgico, producto de la lesión, la hipoxia, la hemorragia, el shock, la manipulación de estructuras, la hipotermia y el dolor. Esta respuesta inflamatoria tiene como objetivo reparar los tejidos dañados e iniciar el proceso de cicatrización. ${ }^{8}$

Se debe considerar que todo procedimiento quirúrgico implica una alta probabilidad de contaminación, debido a la gran cantidad de material producido, como gotas de líquidos biológicos y aerosoles; esto determina que se deban intensificar las medidas de protección en pacientes asintomáticos o negativos para la infección por coronavirus 19 y extremarlas en pacientes con sospecha o confirmación de COVID-19.

Las recomendaciones de las sociedades científicas indican que una máscara facial por sí sola no representa una barrera eficaz para evitar el contagio; esta debe acompañarse del uso de barbijo (tapabocas) N95 y antiparras (gafas) cerradas, sin ventilación para una protección ocular completa. 
Complementariamente, Balibrea y cols. recomiendan que aquellas instituciones que cuenten con varios quirófanos deben reorganizar su distribución y destinar uno de ellos para la intervención quirúrgica de pacientes con COVID-19 confirmado o con alta sospecha de infección. Esto permitiría mantener un circuito de circulación de pacientes y personal independiente de los otros quirófanos, y evitar así la contaminación de espacios y personas.

\section{EVOLUCIÓN POSQUIRÚRGICA}

La evidencia disponible muestra una tasa más alta de internación en Unidades de Terapia Intensiva (UTI) de pacientes operados con COVID-19 confirmado (44\%), que de aquellos internados por la infección que no fueron operados (21\%). El fenómeno se debería a que el estrés quirúrgico coincide con el período de incubación de la enfermedad.

El mayor porcentaje de pacientes que requirieron traslado a una UTI correspondió a adultos mayores, con comorbilidades preexistentes y cirugías más complejas y más prolongadas. Los hallazgos provenientes de instituciones sanitarias del hemisferio norte sugieren que los procedimientos quirúrgicos pueden acelerar y exacerbar la progresión del COVID-19, ya que los pacientes operados desarrollaron síntomas de la infección más precozmente que la población general. Del mismo modo, se han comunicado tasas de mortalidad más altas (hasta 20\%) comparadas con la población COVID-19 positiva no intervenida, en cualquier país del mundo. ${ }^{9}$

\section{RECOMENDACIONES PARA EL MANEJO PREOPERATORIO}

Rodrigues-Pinto y cols. señalan que la práctica ortopédica se ha visto notablemente afectada por la aparición del brote de COVID-19: se han emitido recomendaciones respecto del EPP adecuado para diagnosticar y tratar a pacientes con COVID-19, para médicos especialistas en terapia intensiva, clínica médica y anestesiología, sobre el manejo en salas comunes de internación, en las UTI y las mesas de anestesia. Sin embargo, hasta el momento, hay muy poca información sobre cómo organizar un quirófano para un paciente con COVID-19 y cómo proteger al personal quirúrgico cuando se deben realizar procedimientos impostergables o urgentes. Según la Asociación Española de Cirujanos, estas situaciones requieren adoptar medidas para reducir al mínimo la posibilidad de contagio entre pacientes, la exposición del personal sanitario y el desarrollo de complicaciones posoperatorias. ${ }^{10}$

Chang Liang y cols. mencionan las recomendaciones de varias instituciones europeas durante esta pandemia, las cuales se limitan a plantear que se practiquen solo aquellas intervenciones quirúrgicas de emergencia o urgencia. ${ }^{11}$ Por su parte, Pinto y cols. señalan que, ante la necesidad de operar a pacientes con cuadros impostergables que requieren cirugía ortopédica urgente o temprana, se recomienda realizar cirugías que requieran menos de $24 \mathrm{~h}$ de internación, como procedimientos artroscópicos de cadera, rodilla, tobillo y de partes blandas. Las cirugías programadas, no urgentes que requieren más de $24 \mathrm{~h}$ de internación, en lo posible, deberían postergarse o cancelarse. Las hospitalizaciones más prolongadas (3-5 días) incrementan el riesgo de contagio intrahospitalario y el uso de recursos limitados a la atención médica.

Los médicos especialistas en áreas quirúrgicas deben prestar la máxima atención a la organización y los ajustes preoperatorios, intraoperatorios y posoperatorios para reducir, al mínimo, los riesgos de diseminación intrahospitalaria.

\section{RECOMENDACIONES PREOPERATORIAS}

A continuación, se resumen las recomendaciones de la Asociación Española de Cirujanos y los artículos publicados en la revista Journal of Bone \& Joint Surgery:

- Consensuar con todo el personal interviniente en el ámbito quirúrgico la diagramación del flujo de circulación del personal, los pacientes y el material quirúrgico limpio, estéril, contaminado y altamente contaminado al igual que la limpieza de cada uno de estos, para evitar la propagación intrahospitalaria entre personas. Destinar un quirófano exclusivamente para la atención de pacientes con COVID-19 y así evitar contaminar otros quirófanos.

- Se debe tomar contacto con todos los pacientes el día anterior al procedimiento quirúrgico para indagar si tienen síntomas respiratorios y cualquier factor de riesgo o antecedente de viaje reciente (dentro de los 14 días previos).

- Programar la admisión hospitalaria para el mismo día de la intervención; evitar las admisiones 1 o 2 días antes para cirugías programadas.

- Todos los pacientes que asisten a las clínicas y hospitales serán sometidos a un interrogatorio exhaustivo sobre sus factores de riesgo y a un examen médico de ingreso que incluya la medición de la temperatura comprobada con un escáner térmico. 
- Si es posible, realizar pruebas de detección de coronavirus 19 rutinariamente antes de una intervención quirúrgica urgente o programada.

- A los pacientes con fiebre o síntomas de las vías respiratorias, especialmente aquellos con antecedentes de viaje o contacto positivo, se los remitirá al departamento de emergencias para una evaluación adicional y reducir, al mínimo, la propagación de la enfermedad.

- El día del procedimiento se constatará la temperatura y se verificará la estabilidad de las comorbilidades que presentan.

- Las cirugías no urgentes para pacientes ancianos inmunocomprometidos deberán diferirse hasta un momento oportuno.

- El uso de EPP junto con la indumentaria quirúrgica en cualquier procedimiento considerado de "contacto estrecho", tanto para casos confirmados como para los no confirmados, pues, en una pandemia, debería asumirse que todos los pacientes son positivos.

- Antes de la cirugía, confeccionar una lista del personal mínimo e indispensable para poder realizar la intervención y verificar que se disponga de la cantidad necesaria de EPP. Evitar una exposición innecesaria del personal.

- Es fundamental que todo el equipo quirúrgico haya realizado un entrenamiento previo en la colocación y el retiro supervisado del EPP antes de un procedimiento real.

- Se debe contar con personal entrenado de reserva en caso de que se produzca algún imprevisto, como exceso de calor o circunstancias similares.

- A los equipos médicos de ortopedia se les aconseja usar máscaras quirúrgicas para todos los encuentros con pacientes y seguir prácticas estrictas de higiene de manos.

- Verificar que los EPP (gorras quirúrgicas, gafas protectoras, máscaras N95, batas y guantes quirúrgicos) estén completos y en la cantidad requerida para el procedimiento. Confeccionar una lista de materiales que se necesitarán durante la intervención, y así evitar la circulación del personal desde el quirófano y hacia este.

A fin de disminuir los riesgos de contaminación cruzada, se propone dividir al personal médico del Servicio de Ortopedia y Traumatología para: a) la atención y el seguimiento de pacientes confirmados, b) la atención de urgencias, c) la atención de pacientes ambulatorios, d) la atención en sala de internación y e) la práctica de procedimientos quirúrgicos. Si la cantidad de pacientes confirmados o con alta sospecha aumenta de manera significativa, en la institución, los hospitales deben cerrar sus puertas a las visitas y se cancelarán todas las cirugías programadas. Se permitirán las cirugías ortopédicas solo en casos de traumatismos con lesiones graves y tumores que no responden adecuadamente a la terapia neoadyuvante.

\section{RECOMENDACIONES PARA EL MANEJO INTRAOPERATORIO \\ DEL PACIENTE CON COVID-19 CONFIRMADO O PRESUNTO}

Se informará a todo el personal hospitalario cuál es el quirófano destinado a los pacientes con COVID-19, como así también las recomendaciones y reglamentaciones por cumplir en las instalaciones.

Se recomienda realizar una distribución de las áreas de quirófano, manteniendo una disposición que permita el flujo del personal sanitario para mantener áreas limpias sin contaminación con el coronavirus. Dicha distribución seguirá un orden de crecimiento progresivo y paulatino de las medidas de protección y aislamiento para el personal del quirófano.

Según las recomendaciones publicadas, el flujo de traslado del personal se debe hacer en 5 zonas, respetando estrictamente el uso de cada zona para la función predeterminada de la siguiente manera:

Zona 1: Entrada al vestuario, donde se coloca el EPP básico.

Zona 2: Antesala, donde se realiza la desinfección y se coloca la ropa quirúrgica.

Zona 3: Quirófano (sala para pacientes con COVID-19).

Zona 4: Salida, donde se quita el EPP.

Zona 5: Vestuario de salida, donde se baña el personal.

En los instantes previos a ingresar en la sala de cirugía, se debe verificar que se han mantenido limpios las áreas seguras de circulación del personal y el material quirúrgico requerido. Igualmente, se debe constatar que se cuenta con los EPP necesarios para las personas que participarán en la intervención y que ellos disponen de los materiales indispensables, inclusive mascarillas y gafas quirúrgicas. 
En las puertas de acceso del quirófano, se debe indicar que se está operando a un paciente con COVID-19 confirmado o presunto.

Se debe mantener la menor circulación de personal posible dentro del quirófano, con puertas cerradas, tratando de contar con los materiales necesarios antes de la cirugía. Mantener la menor cantidad de objetos y equipos dentro del quirófano para evitar la contaminación. Todo material no utilizado durante la cirugía debe descartarse al finalizar la operación. ${ }^{12}$

Todos los pacientes que no requieran intubación como parte del procedimiento anestésico deben utilizar barbijo quirúrgico.

Se deben priorizar los procedimientos quirúrgicos breves con abordajes mínimamente invasivos en pacientes con COVID-19 confirmado o presunto, para así disminuir la posibilidad de contagio de los miembros del equipo. Durante las cirugías traumatológicas y ortopédicas, las herramientas eléctricas (electrocauterizadores, sierras óseas y taladros) deben usarse lo menos posible y con su potencia más baja, ya que emiten aerosoles que aumentan el riesgo de propagación del virus.

Durante las operaciones, deben usarse dispositivos de succión (aspiración negativa) para eliminar el humo y los aerosoles que se producen.

A continuación, presentamos una adaptación de los pasos por seguir en las 5 zonas recomendadas en la bibliografía relevada, enfatizando que cada actividad debe respetar el lugar en el que corresponde realizarse. Es importante contar con la supervisión de un compañero al realizar los pasos, con el fin de mantener las extremas medidas de control.

Tabla 1. Recomendaciones para el equipo quirúrgico en la Zona 1, antes de ingresar en el quirófano

\section{Zona 1}

Retirar TODOS los objetos personales y accesorios de los bolsillos de la ropa quirúrgica (reloj, pendientes, anillos, etc.), antes de colocarse el EPP.

Higiene estándar de manos con agua y jabón.

Colocarse botas impermeables y primer gorro (desechable).

Colocarse delantal plomado con su protector tiroideo (opcional, según el tipo de intervención quirúrgica).

Tabla 2. Recomendaciones para el equipo quirúrgico en la Zona 2, antes de ingresar en el quirófano

\section{Zona 2}

Colocarse bata impermeable.

Colocarse mascarilla (tapabocas) FPP2, FPP3 o N95.

Colocarse gafas protectoras cerradas.

Colocarse mascarilla quirúrgica por encima de la primera.

Colocarse un gorro quirúrgico desechable cubriendo el pabellón auricular (sobre el que se ha colocado previamente).

Colocarse pantalla facial.

Lavado de manos quirúrgico según normas generales.

Enfundarse un primer par de guantes estéril.

Higiene quirúrgica de manos por encima de los guantes con producto a base de alcohol.

Colocarse bata quirúrgica habitual (con asistencia para atarla al respaldo).

Colocarse un segundo par de guantes estériles cubriendo los puños. (Se recomienda como opción colocar un tercer par de guantes estéril). 
Tabla 3. Recomendaciones para el equipo quirúrgico en la Zona 3, una vez concluida la intervención quirúrgica Zona 3

Retirar los guantes quirúrgicos, sin tocarlos por fuera (tercer par).

Lavado de los guantes interiores con un producto a base de alcohol (el observador lo irriga sobre los guantes del profesional). Retirar la bata quirúrgica. No tocarla por fuera. Estirarla desde el frontal rompiendo las cintas. Mediante movimientos envolventes plegarla con la parte externa contaminada hacia adentro.

Desechar todo en el contenedor (debe estar a corta distancia), dejándolo caer de manera pausada.

Lavado de los guantes interiores con solución alcohólica (el observador la irriga sobre los guantes del profesional).

Retirar botas impermeables.

Retirar guante quirúrgico (segundo par).

Lavado de manos con producto a base de alcohol.

Retirar la pantalla facial: agachar la cabeza, cerrando ojos y boca. Colocar la pantalla en una bandeja con desinfectante si debe ser reutilizada, o desecharla en un contenedor si es descartable.

Retirar gorro externo y desechar (cerrando ojos y boca).

Lavado de manos con producto a base de alcohol.

Retirar el barbijo quirúrgico. Agachar la cabeza hacia adelante, cerrando ojos y boca. Sujetar las cintas desde atrás y retirar hacia adelante y hacia abajo. No se debe tocar la parte delantera de la mascarilla. Desechar en el contenedor cogiéndola únicamente por las cintas.

Lavado de manos con producto a base de alcohol.

Retirar la bata protectora (secuencia igual a la bata quirúrgica). Desechar en el contenedor (debe estar a corta distancia).

Lavado de manos con producto a base de alcohol.

Retirar los guantes de nitrilo, sin tocarlos por fuera.

Tabla 4. Recomendaciones para el equipo quirúrgico en la Zona 4, luego de salir del área quirúrgica Zona 4

Higiene quirúrgica de manos.

Retirar gafas: agachar la cabeza, cerrando ojos y boca.

Lavado de manos con producto a base de alcohol.

Retirada de mascarilla FPP2, FPP3 o N95.

Tabla 5. Recomendaciones para el equipo quirúrgico en la Zona 5, luego de salir del área quirúrgica Zona 5

Retirar gorro interno.

Lavado de manos con producto a base de alcohol.

Colocar guantes de nitrilo.

Rociar zuecos con solución alcohólica y retirarlos.

Lavado de manos con producto a base de alcohol hasta los codos.

Ducha.

\section{ORDEN DE COLOCACIÓN DEL EPP PARA LA CIRUGÍA SEGÚN RODRIGUES-PINTO}

Entendemos que el uso de un tercer par de guantes estériles dificulta los movimientos finos y delicados, y puede entorpecer momentos de la técnica quirúrgica. Por lo tanto, sería importante usarlos al retirar los elementos de protección quirúrgica. Esta aclaración se debe a que, en este momento, la posibilidad de contacto y contagio es alta. 
En la bibliografía, hay discrepancias respecto del lugar físico donde el personal médico debe colocarse la bata quirúrgica estéril. La Asociación Española de Cirujanos recomienda colocársela dentro del quirófano, mientras que Rodrigues-Pinto y cols. aconsejan colocarse toda la indumentaria estéril en la antesala, para luego ingresar en el quirófano una vez que el paciente esté anestesiado. Esta medida evitaría el desplazamiento del aire y disminuiría la posibilidad de movilización de aerosoles mientras el profesional se viste con la indumentaria quirúrgica dentro del quirófano.

En los pacientes que requieren anestesia general, debe prestarse atención en el momento de la extubación, pues es una de las maniobras más riesgosas, dado que es muy habitual que el paciente tosa y expectore. Se recomienda que el personal médico que realizó la intervención quirúrgica no se haya quitado la indumentaria quirúrgica y el EPP hasta tanto el paciente no haya sido retirado del quirófano (Tablas 1 y 2). ${ }^{13,14}$

\section{ORDEN DEL RETIRO DEL EPP PARA LA CIRUGÍA}

Una vez finalizada la cirugía, es fundamental que el retiro del EPP se haga pausadamente, tomándose los tiempos y los recaudos pertinentes, de a un cirujano por vez, bajo la supervisión de un compañero entrenado, evitando movimientos bruscos, la generación de aerosoles y la posible contaminación de los implementos ubicados debajo del EPP (Tablas 3-5). ${ }^{15}$

\section{RECOMENDACIONES PARA EL MANEJO POSOPERATORIO}

El régimen de visitas a los pacientes que requieran internación prolongada luego de una cirugía debe ser limitado y en un sector/piso independiente de aquellos pacientes con sospecha o diagnóstico de COVID-19. Respecto a estos últimos, deberán estar aislados de acuerdo con los protocolos internacionales y las curaciones intrahospitalarias se deben efectuar adoptando las medidas de protección personal pertinentes. Asimismo, las visitas deben suspenderse, y el paciente únicamente podrá comunicarse por vía telefónica con sus familiares.

El alta hospitalaria de los pacientes operados deberá ser una decisión tomada en forma multidisciplinaria y dependerá tanto de la mejoría clínica (respecto a su infección por coronavirus) como de su evolución posquirúrgica inmediata. En lo que respecta al acto quirúrgico, se priorizará siempre que sea posible otorgar el alta hospitalaria en el menor tiempo posible.

Se deberán explicar, en forma completa y precisa, todas las indicaciones sobre los cuidados de la herida quirúrgica y el plan de manejo medicamentoso, y se hará hincapié en las pautas de alarma que deberá tener el paciente relacionadas con la cirugía a fin de reducir al mínimo y evitar las consultas hospitalarias innecesarias.

Consideramos necesario e importante que cada Servicio planifique y protocolice la terapia de manejo del dolor de acuerdo con la intervención quirúrgica realizada. Se deberá contar, de preferencia, con una medicación basal y una para mitigar el dolor, si es necesario, recordando que es uno de los síntomas más frecuentes en el período posquirúrgico inmediato. Se deberán tener en cuenta las enfermedades concomitantes para adaptar la medicación a la situación clínica particular.

El seguimiento ambulatorio de estos pacientes deberá adaptarse a la complejidad de la cirugía realizada. Si se dispone de un sector independiente intrahospitalario para el cuidado posoperatorio de pacientes con COVID-19 o un centro de atención ambulatoria descentralizado para su manejo, se citará a los pacientes semanalmente para el control evolutivo y la curación de la herida hasta retirar los puntos, y obtener una cicatrización satisfactoria. A partir de ahí, se deben espaciar los controles y los estudios por imágenes de seguimiento solo se realizarán ante la posibilidad de que haya un cambio significativo en la evolución y el manejo clínico. Los pacientes deberán ser examinados en áreas seguras y limpias, usando el EPP básico y la correspondiente higienización de manos con lavados quirúrgicos. Muchos de los hospitales públicos en la Argentina no cuentan con la infraestructura para disponer de un sector independiente para la atención traumatológica posoperatoria de pacientes con COVID-19; por lo tanto, puede ser necesario programar visitas domiciliarias semanales para el control y el cuidado de la herida de estos pacientes en particular, adoptando las medidas de bioseguridad descritas. Se evitaría así el paso del paciente por espacios de circulación pública siguiendo el objetivo de disminuir al mínimo las posibilidades de contagio y, por otro lado, descongestionar el servicio de traslado hospitalario.

Chang Liang y cols. señalan que se debe evaluar el uso de nuevas tecnologías en la institución, como la telemedicina, teleconferencias e iniciativas de telerrehabilitación, que permitan revisar a los pacientes en sus propios hogares, sin someterlos a incómodas visitas hospitalarias y, en consecuencia, disminuir la exposición al posible contagio del COVID-19. 
Como probablemente los servicios de rehabilitación sean muy limitados, deberemos utilizar recursos alternativos con información escrita detallada y plan de ejercicios de rehabilitación domiciliaria adaptados al paciente y con un seguimiento mediante plataformas digitales.

En el aspecto profesional, todo integrante del personal sanitario que haya tenido contacto estrecho con un paciente con infección presunta o confirmada por coronavirus 19 debe comunicarlo inmediatamente al Servicio de Medicina Preventiva/Salud Laboral/Prevención de Riesgos Laborales asociado a su lugar de trabajo.

\section{DISCUSIÓN}

Las constantes modificaciones y recomendaciones de los sistemas sanitarios y gubernamentales debido la alta velocidad de la propagación del COVID-19 generan grandes limitaciones respecto a las conductas por seguir en el sistema de salud y hospitalario. Existen escasos datos fehacientes de pacientes operados durante el período de incubación o la enfermedad. Esto se debería (en el primero de los casos) a la dificultad para el diagnóstico precoz por las características propias de la infección en pacientes asintomáticos y a la falta de pruebas diagnósticas en pacientes sin síntomas, puesto que se ha priorizado testear a quienes desarrollan manifestaciones clínicas y así indicar el correspondiente aislamiento. Asimismo, sostenemos que las medidas de aislamiento con la consiguiente disminución de la circulación poblacional son un recurso que intenta limitar la expansión de la pandemia aplanando su curva de contagio y lograr una menor tasa de infectados con lesiones por accidentes de tránsito que requieran tratamientos de urgencia, como solíamos enfrentar frecuentemente en los días previos al aislamiento social, preventivo y obligatorio en la Argentina. Esto permite que, al disminuir drásticamente el número de pacientes que requieren internación en urgencias de Ortopedia y Traumatología, se pueda planificar un protocolo de circulación intrahospitalario y en el quirófano para el manejo de pacientes con COVID-19 en nuestra especialidad.

A pesar de no ser considerados esenciales en el control de la pandemia del COVID-19, los especialistas en Ortopedia y Traumatología tenemos un papel fundamental en el cambio de conductas sanitario-laborales para mitigar los casos de propagación e infección intrahospitalaria asociadas a procedimientos médicos invasivos. Se deben priorizar los recursos humanos y económicos a la batalla contra el COVID-19 con el objetivo de intentar disminuir el impacto de la enfermedad y así evitar el colapso del sistema de salud y la escasez de insumos sanitarios necesarios para afrontar esta enfermedad. Es por ello que debemos seleccionar/priorizar estrictamente el tipo de intervenciones quirúrgicas, y suspender o cancelar procedimientos no urgentes. Se debe considerar a los pacientes con cuadros no urgentes como candidatos a tratamiento ortopédico, aunque el resultado final no sea el óptimo, identificando aquellos pacientes que puedan requerir una reconstrucción tardía diferida. En estos casos, se proporcionará al paciente la notificación previa correspondiente, dejando asentado en su historia clínica que la decisión médica se toma en el contexto de la pandemia. Proponemos operar a pacientes con cuadros que requieran una resolución urgente en los que esté en riesgo una extremidad o la vida, o que presente enfermedades que pueden provocar secuelas funcionales graves si no se las trata. Es imprescindible indagar profundamente sobre los antecedentes patológicos del paciente, tratando de mitigar las posibles complicaciones posquirúrgicas exacerbadas por el COVID-19. Respecto al equipo quirúrgico, este debe estar compuesto por la menor cantidad de especialistas posibles para lograr la resolución del cuadro por tratar y consideramos que la cirugía debe estar a cargo del cirujano con más experiencia para reducir, al mínimo, el tiempo quirúrgico y las tasas de complicaciones intraquirúrgicas.

Asimismo, es necesario que los especialistas participen activamente en la organización hospitalaria para crear algoritmos terapéuticos y la distribución racional de áreas de trabajo, discriminando las áreas limpias de las contaminadas. Esto último implica diseñar rutas o flujos de circulación del material sanitario, del personal y de los pacientes.

El personal de la salud debe recibir información sobre cómo prevenir la infección y un entrenamiento en el uso adecuado del EPP: cómo colocar y retirar el equipo sin riesgo de contaminar los objetos personales, las manos y la cara.

En la actividad cotidiana, se debe estar alerta, incluso cuando se revisen pacientes de bajo riesgo, ante el alto porcentaje de pacientes asintomáticos circulantes. Es menester defender las prácticas de higiene e incursionar en el desarrollo de las actividades mediadas por nuevas tecnologías en el lugar de trabajo, con el objetivo de disminuir la exposición de los pacientes.

Como médicos y trabajadores de la salud debemos unirnos y desempeñar nuestra función de la mejor manera para superar como sociedad esta pandemia. Un médico enfermo y aislado es un médico que no puede salvar otra vida. Y debemos tener muy en claro que, como especialistas en Ortopedia y Traumatología, podemos hacer más que tratar fracturas: podemos contribuir a salvar vidas. ${ }^{11}$ 


\section{Agradecimientos}

A las comunidades médicas y científicas internacionales por permitir la rápida y fácil divulgación de la información académica actual. Igualmente al personal de salud que brinda su conocimiento y trabajo para mitigar el impacto de la pandemia COVID-19. Finalmente, un especial agradecimiento a nuestras familias por los sacrificios realizados y por el apoyo incondicional durante este difícil momento que estamos atravesando.

Conflicto de intereses: Los autores no declaran conflictos de intereses.

ORCID de A. Cattáneo: https://orcid.org/0000-0001-5254-0760

ORCID de E. F. Coppola: https://orcid.org/0000-0002-2132-7286

ORCID de A. F. Medina: https://orcid.org/0000-0002-5815-3232

\section{BIBLIOGRAFÍA}

1. Organización Mundial de la Salud. Disponible en: https://www.who.int/es/emergencies/diseases/novelcoronavirus-2019/advice-for-public/q-a-coronaviruses. Consulta: 9 de abril, 2020.

2. Balibrea JM, Badia J, Pérez I, Antona E, Peña E, Botella S, et al. Manejo quirúrgico en pacientes con infección por COVID-19. Recomendaciones de la Asociación Española de Cirujanos. Cir Esp 2020. https://doi.org/10.1016/j.ciresp.2020.03.001

3. Wu Z, McGoogan JM. Characteristics of and important lessons from the coronavirus disease 2019 (COVID-19) outbreak in China. JAMA 2020;323(13):1239-42. https://doi.org/10.1001/jama.2020.2648

4. Bai Y, Yao L, Wei T, Tian F, Jin DY, Chen L, et al. Presumed asymptomatic carrier transmission of COVID-19. JAMA 2020 Feb 21. https://doi.org/10.1001/jama.2020.2565

5. Kutihara H, Bisagni P, Faccincani R, Zago M. COVID-19 outbreak in Northern Italy: Viewpoint of the Milan area surgical community. J Trauma Acute Care Surg 2020 Apr 3. http://doi.org/10.1097/TA.0000000000002695

6. National Health Service. Clinical guide for the management of trauma and orthopaedic patients during the coronavirus pandemic. Disponible en: https://www.england.nhs.uk/coronavirus/wp-content/uploads/sites/52/2020/03/ specialty-guide-orthopaedic-trauma-and-coronavirus-v1-16-march-2020.pdf. Consulta: 8 de abril, 2020

7. Amodeo G, Bugada D, Franchi S, Moschetti G, Grimaldi S, Panerai A, et al. Immune function after major surgical interventions: the effect of postoperative pain treatment. J Pain Res 2018;11:1297-1305. https://doi.org/10.2147/JPR.S158230

8. López-Bago A, Gonzáles Reyes RE, Ruíz Santana JE, Rivera Jiménez J. Inmunidad e inflamación en el proceso quirúrgico. Revista de la Facultad de Medicina de la UNAM 2004;61(4):7-15. https://www.medigraphic.com/pdfs/facmed/un-2018/un184b.pdf

9. Lei S, Jiang F, Su W, Chen C, Chen J, Mei W. Clinical characteristics and outcomes of patients undergoing surgeries during the incubation period of COVID-19 infection, EClinicalMedicine 2020. https://doi.org/10.1016/j.eclinm.2020.100331

10. Rodrigues-Pinto R, Sousa R, Oliveira A. The Orthopaedic Forum. Preparing to perform trauma and orthopaedic surgery on patients with COVID-19. J Bone Joint Surg 2020 April 10. https://doi.org/10.2106/JBJS.20.00454

11. Chang Liang Z, Wang W, Murphy D, Po Hui JH. The Orthopaedic Forum Novel coronavirus and orthopaedic surgery. J Bone Joint Surg 2020 Mar 20. http://doi.org/10.2106/JBJS.20.00236

12. Coccolini F, Perrone G, Chiarugi M, Di Marzo F, Ansaloni L, Scandroglio I, et al. Surgery in COVI-19 patients: operational directives. World J Emerg Surg 2020;15:25. https://doi.org/10.1186/s13017-020-00307-2

13. Asociación de Anestesia Analgesia y Reanimación de Buenos Aires. Recomendaciones para el manejo de la vía aérea en pacientes infectados o con sospecha de infección por coronavirus COVID-19. Disponible en: https://www.aaarba.org/Seccion/coronavirus_29

14. Ti LK, Ang LS, Foong TW, Ng BSW. What we do when a COVID-19 patient needs an operation: operating room preparation and guidance. Can J Anesth 2020 Mar 6. https://doi.org/10.1007/s12630-020-01617-4

15. Universidad Nacional de La Plata. Coronavirus (COVID-19). Secuencia de procedimientos de bioseguridad para equipos de salud. Disponible en: https://unlp.edu.ar/coronavirus-salud 